\title{
Third-order nonlinear phenomenon generated on the inner surface of bulk lithium niobate crystals with magnesium doping
}

\author{
Xiaojing Wang (王晓静) $)^{1^{*}}$, Xiaobo Li (李晓波) ${ }^{1}$, Hui Xu (许 辉) ${ }^{1}$, Longhui He (贺龙辉) ${ }^{1}$, Xuelei Li (李雪垒) ${ }^{1}$ ， \\ Yulan Dong (董玉兰) $)^{1}$, and Xianfeng Chen (陈险峰) $)^{2,3}$ \\ ${ }^{1}$ Department of Applied Physics, Microelectronic and Physics, Hunan University of Technology and Business, Changsha 410205, China \\ ${ }^{2}$ Department of Physics and Astronomy, Shanghai Jiao Tong University, Shanghai 200240, China \\ 3 Key Laboratory for Laser Plasma (Ministry of Education], IFSA Collaborative Innovation Center, Shanghai Jiao Tong University, Shanghai 200240, China
}

*Corresponding author: 1029125891@qq.com

Received November 5, 2021 | Accepted December 2, 2021 | Posted Online January 10, 2022

\begin{abstract}
When two synchronized laser beams illuminate the inner surface of bulk lithium niobate crystals with magnesium doping

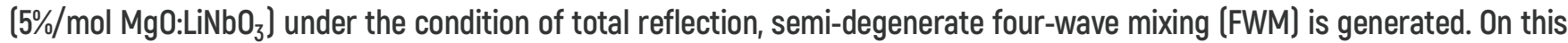
basis, a more sophisticated frequency conversion process on the interface of nonlinear crystal has been researched. The generation mechanism of FWM is associated with the fundamental waves reflected on the inner surface of the nonlinear crystal. Analysis of the phase-matching mechanism confirms that the FWM is radiated by the third-order nonlinear polarized waves, which are stimulated by the third-order nonlinear susceptibility coefficient of the nonlinear crystal. Theoretically calculated and experimentally measured corresponding data have been presented in this article. These results are expected to provide new inspiration for further experimental and theoretical research on frequency conversion in nonlinear crystals.
\end{abstract}

Keywords: nonlinear optics; four-wave mixing; surface of nonlinear crystals; third-order nonlinear coefficient. DOI: 10.3788/COL202220.031901

\section{Introduction}

Researchers pay more and more attention to the investigation of low-dimensional nonlinear processes, such as material physical interface, artificial polarized crystal domain walls ${ }^{[1]}$, and bonding or coating interface of different nonlinear materials ${ }^{[2]}$. The existence of discrete particles in the interface of nonlinear material ${ }^{[3]}$ has significant impact on the nonlinear frequency conversion. Many second-order nonlinear frequency conversion effects at the interface of nonlinear crystals have been studied extensively. What is more, ultra-short pulses of light can also be observed through the stimulation of sum frequency generated on the domain walls of periodically polarized crystals ${ }^{[4-6]}$. Nonlinear Cherenkov cascading difference frequency ${ }^{[7]}$, third harmonics, and other higher harmonics ${ }^{[8,9]}$ generated in the superlattice structure have also been the extensive subject of numerous investigations.

Due to the perfectly phase-matching mechanism, the conversion efficiency of the second harmonic generated on the inner surface of the crystal is relatively high ${ }^{[10-12]}$. The efficiency of nonlinear Cherenkov doubling frequency and sum frequency conversion generated on the crystal surface have also been enhanced $^{[11-21]}$. Although second-order nonlinear processes on surfaces and interfaces have been extensively studied, thirdorder nonlinear processes are rarely studied. The phasematched nonlinear Cherenkov difference frequency emitted on the crystal interface also can generate effective terahertz waves ${ }^{[22,23]}$. So, the study of third-order nonlinear effects on crystal surfaces and interfaces has extensive application value. Four-wave mixing (FWM) is a nonlinear optical phenomenon caused by the interaction of four coherent waves through third-order nonlinear susceptibility coefficients of the nonlinear crystal. Conventionally, the third-order nonlinear susceptibility is relatively small, so efficient FWM processes require long interaction length. Since efficient optical frequency conversion requires coherent accumulation of the nonlinear harmonics wave of a single atom, appropriate phase matching between the fundamental and harmonic waves must be satisfied. In the FWM process, phase-matching mechanisms are determined according to the propagation direction of fundamental waves and experimental conditions.

In this article, we experimentally demonstrate an innovative FWM process generated on the boundary of bulk lithium niobate crystals with magnesium doping for the first time, to the 
best of our knowledge. The reflected light of the fundamental wave on the boundary also participates in the generation of this FWM. The random scattering light of the fundamental wave also participates in the generation of the ring FWM in this experiment. The FWM is widely used in the areas of medical science, including optical processing ${ }^{[24]}$, nonlinear imaging, real-time holography, phase conjugate optics, and entangled photon pair production $^{[25-30]}$. The optical characteristics of the more complicated laser frequency conversion process in surface nonlinear crystal $^{[31-33]}$ need to be deeply studied.

\section{Experiment}

Figure 1(a) illustrates the experimental arrangement. The experimental layout is shown in Fig. 1(a). In our experiment, the pump beam is produced by a Ti:sapphire femtosecond laser source with the pulse duration of 75 fs and central wavelength of $800 \mathrm{~nm}$. The probe beam with the wavelength of $1300 \mathrm{~nm}$ is also provided by the optical parametric amplifier. We use a pump wave centered at $800 \mathrm{~nm}$ and a signal wave centered at $1300 \mathrm{~nm}$ (both extraordinarily polarized) to illuminate the surface of the crystal as a fundamental frequency wave. The crystal is a $z$-cut $5 \% / \mathrm{mol} \mathrm{MgO}: \mathrm{LiNbO}_{3}$ of the size $3 \mathrm{~mm} \times 10 \mathrm{~mm} \times$ $2 \mathrm{~mm}(X \times Y \times Z)$. All of surfaces are polished. When two synchronized laser beams horizontally or obliquely illuminate the boundary of the crystal at the angle of $\alpha$ with respect to the $x$ axis, a series of colorful laser beams appear on the back plate, as shown in Figs. 1(b) and 1(c), respectively.

The centric two SHG spots, which are marked spot 0 [as shown in Fig. 1(c)], are overlapped in space. They are the non-collinear phase-mismatched SHG, which are generated by the sum frequency polarization waves. They are stimulated by the incident and reflected waves of two fundamental waves at $1300 \mathrm{~nm}$ and $800 \mathrm{~nm}$, respectively. Spot 1 in Fig. 1(c) represents two collinear phase-mismatched SHG beams, which are overlapped in space. They are generated by the reflected wave

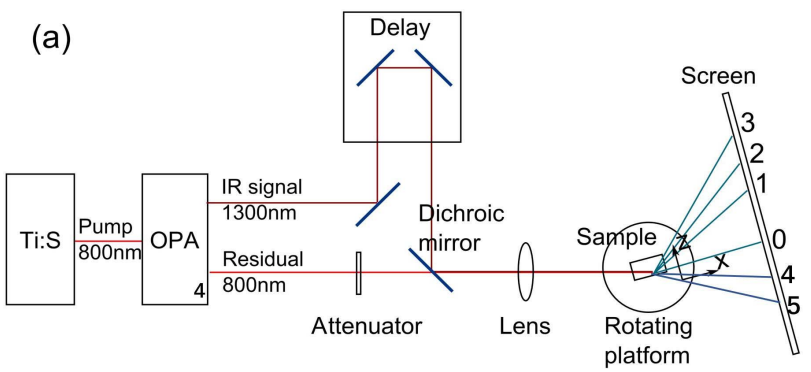

(b)

(c)
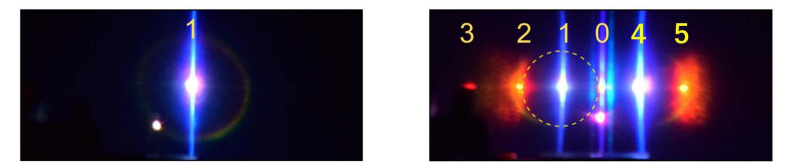

Fig. 1. (a) Experimental setup. (b) and (c) The FWM changing process of the experimental phenomenon when rotating the nonlinear crystal on the experiment stage. of the two fundamental waves with wavelengths of $800 \mathrm{~nm}$ and $1300 \mathrm{~nm}$, respectively. The red spot marked 3 in Fig. 1(c) is the nonlinear Cherenkov radiation of the fundamental wave of $1300 \mathrm{~nm}$. The spots marked 4 and 5 in Fig. 1(c) are the transmitted light of points 1 and 2, respectively. Spots 4 and 5 are symmetrical with points 1 and 2 about point 0 , respectively. The specific phase-matching mechanisms about spots 0,1 , and 3 are explained in our previous article on interface second harmonic phenomenon ${ }^{[11,12]}$. Our present research focuses on the generation mechanism of the yellow spot marked 2 in Fig. 1(c). We observe that the intensity of the FWM achieves the highest at a special incident angle.

The position of the yellow spot marked 2 in Fig. 1(c) is between spot 3 and spot 1 . When the nonlinear crystal is rotated to increase the angle between the incident light and the boundary of the crystal, the angle of the emitted yellow spot 2 increases. Since the spectrum of the incident femtosecond laser is broadband, the center wavelength of spot 2 changes from $617.9 \mathrm{~nm}$ to $545.8 \mathrm{~nm}$ with different incident angles from $10^{\circ}$ to $16^{\circ}$, respectively. Spot 2 is extraordinarily polarized through experimental verification.

\section{Theoretical Discussion}

There are three possibilities for the formation of the yellow spot marked 2 in Fig. 1(c). The potential phase-matching geometries are demonstrated in Fig. 2(a), respectively. Their phase-matching wave vector equations are demonstrated in Eqs. (1)-(3), respectively. Equation (1) demonstrates that the $800 \mathrm{~nm}$ fundamental wave generates $400 \mathrm{~nm}$ second harmonics, then the generated $400 \mathrm{~nm}$ harmonics wave cascades with the $1300 \mathrm{~nm}$ fundamental wave to generate difference frequency $578 \mathrm{~nm}$ light. Equation (2) demonstrates that two fundamental waves at $1300 \mathrm{~nm}$ and $800 \mathrm{~nm}$ generate difference frequency of $2080 \mathrm{~nm}$, and then the difference frequency of $2080 \mathrm{~nm}$ cascades with the fundamental wave at $800 \mathrm{~nm}$ to generate the sum frequency wave at $578 \mathrm{~nm}$. Both methods are cascaded secondorder nonlinear optical parametric processes. The third
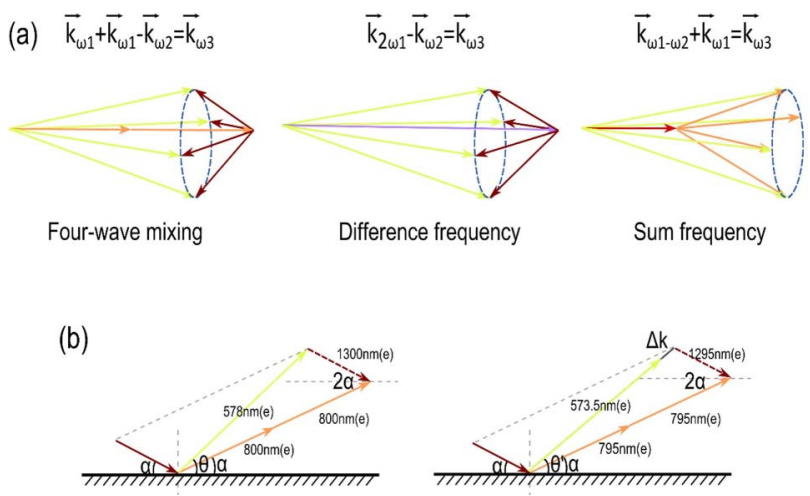

Fig. 2. (a) Three-dimensional phase-matching geometry of the FWM, difference frequency, and sum frequency assisted by the scattered light. (b) The perfect phase-matching geometry of FWM in the horizontal plane. 
possibility is through FWM. Under this condition, the fundamental wave at $800 \mathrm{~nm}$ and $1300 \mathrm{~nm}$ generates the $578 \mathrm{~nm}$ wave directly, as shown in Eq. (3):

$$
\begin{gathered}
\hbar \omega_{800 \mathrm{~nm}}+\hbar \omega_{800 \mathrm{~nm}}=\hbar \omega_{400 \mathrm{~nm}} \\
\hbar \omega_{400 \mathrm{~nm}}-\hbar \omega_{1300 \mathrm{~nm}}=\hbar \omega_{578 \mathrm{~nm}} \\
\hbar \omega_{800 \mathrm{~nm}}-\hbar \omega_{1300 \mathrm{~nm}}=\hbar \omega_{2080 \mathrm{~nm}} \\
\hbar \omega_{2080 \mathrm{~nm}}+\hbar \omega_{800 \mathrm{~nm}}=\hbar \omega_{578 \mathrm{~nm}} \\
\hbar \omega_{800 \mathrm{~nm}}+\hbar \omega_{800 \mathrm{~nm}}-\hbar \omega_{1300 \mathrm{~nm}}=\hbar \omega_{578 \mathrm{~nm}}
\end{gathered}
$$

In order to clarify the generation mechanism of the $578 \mathrm{~nm}$ wave, making a concrete analysis is needed. By calculating the e-polarized refraction index of the interactive light with wavelengths of $1300 \mathrm{~nm}, 578 \mathrm{~nm}$, and $400 \mathrm{~nm}$, we find that their wave vectors do not satisfy the condition of the cascaded secondorder nonlinear optical parametric processes, because the wave vector of the $400 \mathrm{~nm}$ wave is larger than the sum of the $578 \mathrm{~nm}$ and $1300 \mathrm{~nm}$ waves. That means the inequality $\left|\hbar \vec{k}_{400 \mathrm{~nm}}\right|>$ $\left|\hbar \vec{k}_{1300 \mathrm{~nm}}\right|+\left|\hbar \vec{k}_{578 \mathrm{~nm}}\right|$ is always satisfied. So, the triangle phase-matching condition cannot be satisfied. Thus, the possibility of cascaded difference frequency is excluded. For the third-order nonlinear optical parametric process, the inequality $\left|\hbar \vec{k}_{800 \mathrm{~nm}}\right|+\left|\hbar \vec{k}_{800 \mathrm{~nm}}\right|+\left|\hbar \vec{k}_{1300 \mathrm{~nm}}\right|>\left|\hbar \vec{k}_{578 \mathrm{~nm}}\right|$ is always satisfied. So, the third-order nonlinear optical parametric process is the reason for generation of the yellow spot at $578 \mathrm{~nm}$.

Because the fundamental waves have different frequency components, when the fundamental frequency light with a special wavelength illuminates the crystal interface at the corresponding incident angle, the maximum conversion efficiency of FWM appears. For the incident angle corresponding to certain fundamental frequency waves, the wave vector relationship of the phase-mismatched configuration can be expressed as

$$
\begin{aligned}
\Delta k & =\vec{k}_{1}+\vec{k}_{1}-\vec{k}_{2}-\vec{k}_{\mathrm{FWM}} \\
& =\sqrt{4 k_{1}^{2}(\alpha)+k_{2}^{2}(\alpha)-4 k_{1}(\alpha) k_{2}(\alpha) \cos 2 \alpha}-k_{\mathrm{FWM}}(\theta),
\end{aligned}
$$

where $\vec{k}_{1}, \vec{k}_{2}$, and $\vec{k}_{\mathrm{FWM}}$ represent wave vectors of $800 \mathrm{~nm}$, $1300 \mathrm{~nm}$, and $578 \mathrm{~nm}$, respectively, and the emission angle $\theta$ of FWM satisfies the equation $\tan \theta=\tan \alpha \frac{2 k_{1}(\alpha)+k_{2}(\alpha)}{2 k_{1}(\alpha)-k_{2}(\alpha)}$. Nonlinear polarized waves stimulated by three fundamental waves can be expressed as

$$
P_{\mathrm{NL}}^{(3)}\left(\omega_{\mathrm{FWM}}\right)=\varepsilon_{0} \chi_{s}^{(3)}\left(-\omega_{\mathrm{FWM}} \vdots \omega_{1}, \omega_{1},-\omega_{2}\right) \vdots \vec{E}_{1} \vec{E}_{1} \vec{E}_{2}^{*} .
$$

The frequency spectra of incident fundamental light at $800 \mathrm{~nm}$ and $1300 \mathrm{~nm}$ are standard Gaussian distributions, and their spectral bandwidths (FWHM) are $40 \mathrm{~nm}$ and $65 \mathrm{~nm}$, respectively. The electric field vector of the fundamental wave is the $x-z$ plane, and $\chi_{s}^{(3)}$ is the third-order nonlinear coefficient of the crystal. The theoretically calculated results of the third-order nonlinear coefficient of bulk lithium niobate crystals with magnesium doping crystals are $4.96 \times 10^{-13}$ esu in this experiment. The waist diameter of the incident fundamental light is about $40 \mu \mathrm{m}$, and the internal incidence angle of the fundamental frequency wave is about $5.5^{\circ}$. Figure $2(\mathrm{~b})$ shows the perfect phasematching geometry of FWM [point 2 in Fig. 1(c)] in the horizontal plane. The fundamental wave that participates in the phase-matching of FWM is the reflected light at $1300 \mathrm{~nm}$ rather than scattered light of $1300 \mathrm{~nm}$. Since the intensity of reflected light is much stronger than that of scattered light, the intensity of FWM is greatly enhanced at an incident angle of the fundamental wave. The complete phase-matching relationship of this wave vector is demonstrated as

$$
\begin{aligned}
& k_{1300}^{2}(\alpha)+4 k_{800}^{2}(\alpha)-4 k_{800}(\alpha) k_{1300}(\alpha) \cos 2 \alpha=k_{578}^{2}(\theta), \\
& \frac{k_{1300}(\alpha)}{\sin (\theta-\alpha)}=\frac{k_{578}(\theta)}{\sin 2 \alpha} .
\end{aligned}
$$

Equation (6) indicates that only when the incident angle reaches a specific angle $\alpha$, perfect phase matching of FWM is generated. That means when synchronously collinear fundamental waves illuminate on the crystal interface with angle $\alpha$, the corresponding radiated angle $\theta$ of FWM is also determined. At this time, the conversion efficiency of FWM reaches the maximum:

$$
\left\{\begin{array}{l}
2 \frac{n_{1}(\alpha)}{\lambda_{1}} \sin \alpha+\frac{n_{2}(\alpha)}{\lambda_{2}} \sin \alpha=\frac{n_{\mathrm{FWM}}(\theta)}{\lambda_{\mathrm{FWM}}} \sin \theta \\
2 \frac{n_{1}(\alpha)}{\lambda_{1}} \cos \alpha-\frac{n_{2}(\alpha)}{\lambda_{2}} \cos \alpha=\frac{n_{\mathrm{FWM}}(\theta)}{\lambda_{\mathrm{FWM}}} \cos \theta
\end{array},\right.
$$

or the relationship of the wave vector with the angle demonstrated in Eq. (6) can be expressed in the formation of wavelength in Eq. (7), where the internal angle of the fundamental wave with the boundary of the nonlinear crystal is $\alpha$, and the internal emitted angle of FWM is $\theta . \lambda_{1}$ and $\lambda_{2}$ are the wavelengths of the two fundamental frequencies in the vacuum. $n_{1}, n_{2}$, and $n_{\mathrm{FWM}}$ are the refractive indices of extraordinary light corresponding to the two fundamental waves and FWM in their propagation direction, as demonstrated in Eq. (7). It can be seen that their numerical value varies with corresponding angles, respectively.

By solving these two equations, the relationship between emission angles of FWM and the incidence angles of fundamental frequency waves on the surface of the crystal can be calculated. Equation (6) indicates that when the fundamental frequency waves with specific wavelengths are incident to the surface of the nonlinear crystal, perfect phase-matching FWM is radiated only when incident angles reach corresponding data. The conversion efficiency of FWM reaches the maximum at the same time. Because the relationship between emergence angles of FWM and incidental angles of the fundamental wave in Eq. (6) is complicated, it is impossible to get a continuously smooth curve. But, a series of separated spots can be 

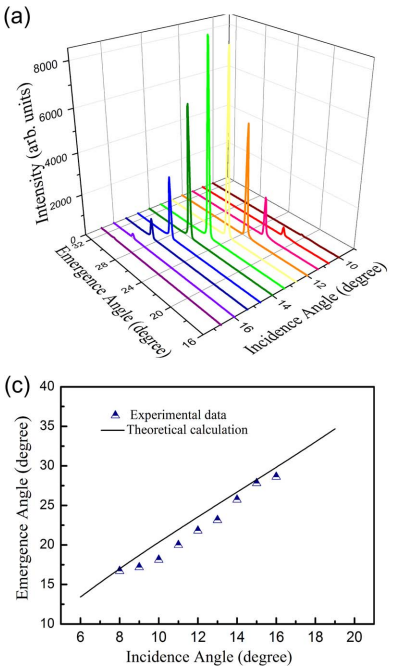

(b)
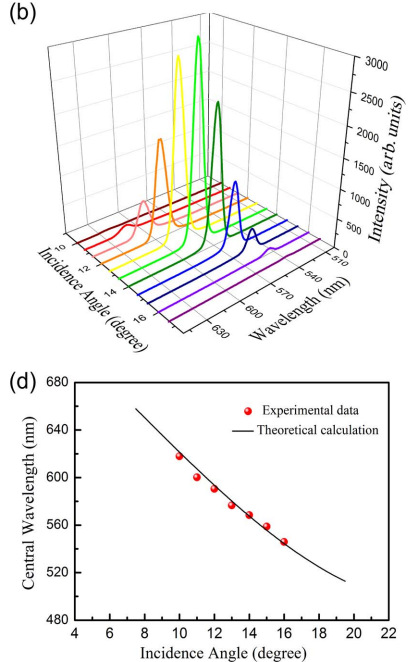

Fig. 3. (a) Light intensity of fundamental frequency wave varying with the radiated angle of FWM and the incident angle of fundamental frequency wave. (b) Light intensity of FWM varying with the central wavelength of FWM and the incident angle of fundamental frequency wave. (c) Theoretically calculated and experimentally measured emergence angles of FWM at different incidence angles of fundamental waves. (d) Theoretically calculated and experimentally measured central wavelengths of FWM at different incidence angles of fundamental waves.

theoretically calculated, as shown in Fig. 3(c). In this experiment, the phase-matching angle of FWM has a wide line-width range.

Furthermore, the relationship among radiated angles of FWM, light intensity of fundamental frequency waves, and incident angles of fundamental frequency waves is shown in Fig. 3(a). It is known that FWM has a spatial distribution of about $0.3^{\circ}$. The relationship among the central wavelength and light intensity of FWM, and incident angles of fundamental frequency waves is shown in Fig. 3(b). The theoretically calculated and experimentally measured data of radiation angles and central wavelength of FWM corresponding to different incident angles of specific fundamental frequency waves are shown in Tables 1 and 2, respectively. It should be noted that the experimentally measured spectral width is about $3 \mathrm{~nm}$, and the spectral width of FWM is between $9 \mathrm{~nm}$ and $11 \mathrm{~nm}$ through calculation in Fig. 3(b). This is because the calculated spectral width includes the spectral width of FWM that is radiated at all angles. Experimentally measured data only include the spectral width at the position where FWM intensity is maximum.

(a)
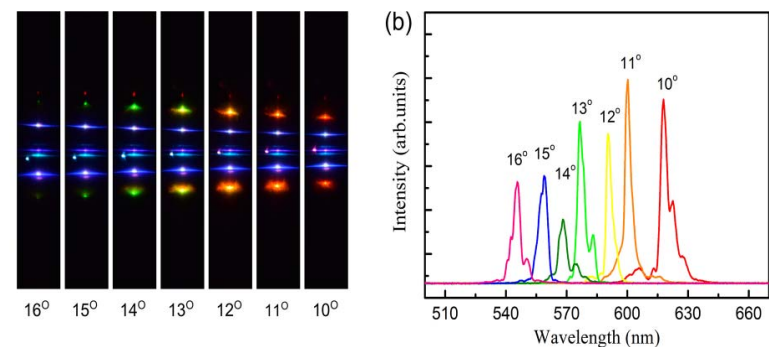

Fig. 4. (a) Photographs of FWM signals with different incidence angles of fundamental waves. (b) Spectra of FWM signals with different incidence angles.

Due to the small divergence angle of the spectrometer, the experimentally measured spectral width is smaller than the calculated width.

Because the fundamental frequency waves are extraordinarily polarized light in the nonlinear conversion process, the nonlinear bulk crystal is $5 \% / \mathrm{mol} \mathrm{MgO}: \mathrm{LiNbO}_{3}$. The third-order nonlinear coefficient is $4.96 \times 10^{-13}$ esu in this experiment. Light intensities of fundamental frequency waves and FWM are experimentally measured, as demonstrated in Figs. 3(a) and 3(b). When the incidence angle of the fundamental frequency wave is $13.4^{\circ}$, the emergency angle of FWM is $25.18^{\circ}$, and the light intensities of fundamental frequency waves and FWM are $7960 I_{0}$ and $2610 I_{0}$, respectively. $I_{0}$ is the relative standard unit of intensity. The experimentally measured conversion efficiency of FWM is $32 \%$. The interactive length is about $0.13 \mathrm{~mm}$. Furthermore, theoretically calculated and experimentally measured emergence angles of FWM corresponding to different incidence angles of fundamental waves are displayed in Fig. 3(c). Theoretically calculated and experimentally measured central wavelengths of FWM at different incidence angles of fundamental waves are shown in Fig. 3(d).

The experimentally measured and theoretically calculated data agree with each other very well in Figs. 3(a)-3(d). In this experiment, the spectrum of phase-matching FWM has a large bandwidth. The central wavelength of FWM changes from $620 \mathrm{~nm}$ to $545 \mathrm{~nm}$ with corresponding incident angles of fundamental waves, as shown in Figs. 4(a) and 4(b).

The reason for the large bandwidth of FWM is that the incident light is a femtosecond laser, so the fundamental frequency spectrum has a broad bandwidth to stimulate the FWM with a large bandwidth. When different incidence angles of fundamental waves satisfy corresponding data of phase matching for different frequency components in fundamental

Table 1. Theoretically Calculated and Experimentally Measured Data of Emergence Angle of FWM Corresponding to Different Incidence Angles of Specific Fundamental Frequency Waves.

\begin{tabular}{|c|c|c|c|c|c|c|c|c|c|}
\hline Incidence angle of fundamental wave $\left({ }^{\circ}\right)$ & 8 & 9 & 10 & 11 & 12 & 13 & 14 & 15 & 16 \\
\hline Experimentally measured emergence angle $\left({ }^{\circ}\right)$ & 16.67 & 17.13 & 18.09 & 19.97 & 21.78 & 23.11 & 25.68 & 27.76 & 28.55 \\
\hline
\end{tabular}


Table 2. Theoretically Calculated and Experimentally Measured Data of Central Wavelength of FWM Corresponding to Different Incidence Angles of Specific Fundamental Frequency Waves.

\begin{tabular}{|c|c|c|c|c|c|c|c|}
\hline Incidence angle of fundamental wave $\left({ }^{\circ}\right)$ & 10 & 11 & 12 & 13 & 14 & 15 & 16 \\
\hline Experimentally measured central wavelength (nm) & 617 & 598 & 590 & 576 & 568 & 558 & 545 \\
\hline
\end{tabular}

waves, specific radiated angles of FWM appear, as shown in Figs. 4(a) and 4(b). This is the reason why the central wavelength of the FWM changes with different incidence angles of fundamental waves. The FWM has certain spatial distributions, so a small phase mismatch is allowed.

\section{Conclusion}

In summary, a new method to generate FWM stimulated by two synchronized laser beams on the interface with $0-1$ abrupt change of a nonlinear optical susceptibility coefficient is demonstrated, in addition to the waveguide and the domain wall structure. Experimentally measured data and theoretically calculated data fit well with each other. Then, phase-matching geometry of the FWM is analyzed. This novel experiment method may have a broad range of applications, such as dark-field microscopy, frequency selective excitation of surface modes, surface plasmon polaritons, guided waveguide modes, and surface phonon polaritons.

\section{Acknowledgement}

This work was supported by the National Natural Science Foundation of China (Nos. 61125503, 61235009, and 61801520), the Foundation for Development of Science and Technology of Shanghai (No. 13JC1408300), the Natural Science Foundation of Hunan Province (Nos. 2019JJ50756 and 2018JJ3521), and the Scientific Research Project of Hunan Province Education Department (No. 20B144).

\section{Data Availability}

The data that support the findings of this study are available from the corresponding author upon reasonable request.

\section{References}

1. H. Ren, X. Deng, Y. Zheng, and X. Chen, "Enhanced nonlinear Cherenkov radiation on the crystal boundary," Opt. Lett. 38, 1993 (2013).

2. S. Palomba and L. Novotny, "Nonlinear excitation of surface plasmon polaritons by four-wave mixing," Phys. Rev. Lett. 101, 056802 (2008).

3. M. Lippitz, M. A. van Dijk, and M. Orrit, "Third-harmonic generation from single gold nanoparticles," Nano Lett. 5, 799 (2005).

4. A. Fragemann, V. Pasiskevicius, and F. Laurell, "Second-order nonlinearities in the domain walls of periodically poled $\mathrm{KTiOPO}_{4}$," Appl. Phys. Lett. 85, 375 (2004).
5. S. J. Holmgren, C. Canalias, and V. Pasiskevicius, "Ultrashort single-shot pulse characterization with high spatial resolution using localized nonlinearities in ferroelectric domain walls," Opt. Lett. 32, 1545 (2007).

6. J. Trull, I. Sola, and B. Wang, "Ultrashort pulse chirp measurement via transverse second-harmonic generation in strontium barium niobate crystal," Appl. Phys. Lett. 106, 221108 (2015).

7. R. Ni, L. Du, and Y. Wu, "Nonlinear Cherenkov difference-frequency generation exploiting birefringence of KTP," Appl. Phys. Lett. 108, 031104 (2016).

8. C. Chen, J. Lu, and Y. Liu, "Cerenkov third-harmonic generation via cascaded $\chi^{(2)}$ processes in a periodic-poled $\mathrm{LiTaO}_{3}$ waveguide," Opt. Lett. 36, 1227 (2011).

9. H. X. Li, S. Y. Mu, and P. Xu, "Multicolor Cherenkov conical beams generation by cascaded- $\chi^{(2)}$ processes in radially poled nonlinear photonic crystals," Appl. Phys. Lett. 100, 101101 (2012).

10. H. Ren, X. Deng, Y. Zheng, and X. Chen, "Surface phase-matched harmonic enhancement in a bulk anomalous dispersion medium," Appl. Phys. Lett. 103, 021110 (2013).

11. X. Wang, H. Ren, N. An, X. Zhao, Y. Zheng, and X. Chen, "Large acceptance of non-collinear phase-matching second harmonic generation on the surface of an anomalous-like bulk dispersion medium," Opt. Express 22, 28234 (2014).

12. X. Wang, J. Cao, X. Zhao, and X. Chen, "Sum-frequency nonlinear Cherenkov radiation generated on the boundary of bulk medium crystal," Opt. Express 23, 31838 (2015).

13. P. Morin, J. Fatome, C. Finot, S. Pitois, R. Claveau, and G. Millot, "All-optical nonlinear processing of both polarization state and intensity profile for $40 \mathrm{Gbit} / \mathrm{s}$ regeneration applications," Opt. Express 19, 17158 (2011).

14. B. Haji-Saeed, S. K. Sengupta, M. Testorf, W. Goodhue, J. Khoury, C. L. Woods, and J. Kierstead, "Real-time holographic deconvolution techniques for one-way image transmission through an aberrating medium: characterization, modeling, and measurements," Appl. Opt. 45, 3298 (2006).

15. C. J. McKinstrie, S. Radic, and C. Xie, "Reduction of soliton phase jitter by in-line phase conjugation," Opt. Lett. 28, 1519 (2003).

16. J. E. Sharping, K. F. Lee, M. A. Foster, A. C. Turner, B. S. Schmidt, M. Lipson, A. L. Gaeta, and P. Kumar, "Generation of correlated photons in nanoscale silicon waveguides," Opt. Express 14, 12388 (2006).

17. Z. Xu, B. Luo, Y. Chen, and X. Li, "High sensitivity and anisotropic broadband photo response of Td-WTe 2 ," Phys. Lett. A 389, 127093 (2021).

18. N. An, H. Ren, Y. Zheng, and X. Chen, "Cherenkov high-order harmonic generation by multistep cascading in $\chi^{(2)}$ nonlinear photonic crystal," Appl. Phys. Lett. 100, 221103 (2012).

19. S. Tang, X. Li, X. Xiao, and X. Zhang, "Effects of $\mathrm{NH}_{3}$ flow rate on the growth mechanism and optical properties of InN crystallites fabricated by chemical vapor deposition," Cryst. Growth Des. 20, 4928 (2020).

20. T. Li, X. Zhao, Y. Zheng, and X. Chen, "Conical second harmonic generation in KDP crystal assisted by optical elastic scattering," Opt. Express 23, 23827 (2015).

21. N. An, Y. Zheng, H. Ren, and X. Chen, "Conical second harmonic generation in one-dimension nonlinear photonic crystal," Appl. Phys. Lett. 102, 201112 (2013).

22. K. Suizu, T. Akiba, N. Kaneko, H. Uchida, K. Miyamoto, and T. Omatsu, "Cherenkov phase-matched terahertz wave generation and its spectroscopic applications," Proc. SPIE 8909, 890910 (2013).

23. H. Xu, Z. He, Z. Chen, G. Nie, and H. Li, "Optical Fermi level-tuned plasmonic coupling in a grating-assisted graphene nanoribbon system," Opt. Express 28, 25767 (2020). 
24. Z. Chen, S. Zhang, Y. Chen, Y. Liu, P. Li, Z. Wang, X. Zhu, K. Bi, and H. Duan, "Double Fano resonances in hybrid disk/rod artificial plasmonic molecules based on dipole-quadrupole coupling," Nanoscaled 12, 9776 (2020).

25. H. Huang, S. Wang, X. Liu, and D. Shen, "Generation of 100kw narrow-line far-infrared radiation from a KTP off-axis THZ parametric oscillator," Phys. Technol. 93, 91 (2018).

26. G. Schunemann, T. Zawilski, A. Pomeranz, J. Creeden, and A. Budni, "Advances in nonlinear optical crystals for mid-infrared coherent sources," Opt. Phys. 11, 36 (2016).

27. L. Li, H. Liu, and X. Chen, "Dynamic manipulation of nonlinear Talbot effect with structured light," Opt. Lett. 46, 1281 (2021).

28. A. Bakman, S. Fishman, M. Fink, E. Fort, and S. Wildeman, "Observation of the Talbot effect with water waves," Am. J. Phys. 87, 38 (2019).
29. L. Urbanski, A. Isoyan, A. Stein, J. J. Rocca, C. S. Menoni, and M. C. Marconi, "Defect-tolerant extreme ultraviolet nanoscale printing," Opt. Lett. 37, 3633 (2012).

30. Y. Sheng, A. Best, H. J. Butt, W. Krolikowski, A. Arie, and K. Koynov, “Three dimensional ferroelectric domain visualization by Čerenkov-type second harmonic generation," Opt. Express 18, 16539 (2010).

31. Y. Fang, J. Cheng, S. Wang, C. Gu, and L. Xu, "Design and analysis of a rangeextended acidity detector based on a Nile red laser with tandem cuvette system," Chin. Opt. Lett. 18, 111402 (2020).

32. Y. Liu, T.-J. Wang, N. Chen, H. Guo, and R. Li, "Simultaneous generation of controllable double white light lasers by focusing an intense femtosecond laser pulse in air," Chin. Opt. Lett. 18, 121402 (2020).

33. C. Liu, J. Lü, W. Liu, F. Wang, and P. K. Chu, "Overview of refractive index sensors comprising photonic crystal fibers based on the surface plasmon resonance effect," Chin. Opt. Lett. 19, 102202 (2021). 